\title{
Research on 3-D Trajectory Prediction
}

\author{
Yubing Dong1, a, Ying Sun2, b, Mingjing Li1,c \\ 1College of Electronics and Information Engineering, Changchun \\ University, \\ Changchun 130022, China \\ aemail:dyblbq@126.com, bemail:522929975@qq.com, cemail: \\ Imj5320510@163.com
}

\begin{abstract}
The innovation of this paper is that a mathematical model of 3-D trajectory prediction is proposed and studied, based on 2-D trajectory prediction algorithm. And 3-D trajectory prediction algorithm is applied to object tracking system, in order to fast and efficient tracking. A large number of experiments are done by MALTAB. By comparing the test results, prediction trajectory is consistent with actual trajectory.

Keywords: 3-D Trajectory Prediction; 2-D Trajectory Prediction; Object Tracking; Algorithm

\section{Introduction}

The real-time detection algorithm of the moving point target in image sequences is one of the key algorithms in many real-time processing systems. There are many challenges for a long precise tracking of target, because the target status and the surrounding environment changes over time, such as target posture, illumination changes, occlusion, frame loss, etc. Therefore, to seek low cost, high precision and real time calculation method of target motion trajectory has become a focus in research of target tracking. In order to solve the above problems, a method based on the trajectory estimation algorithm for moving target tracking is proposed. Trajectory prediction algorithm is widely used in many aspects such as computer vision, automatic traffic, human detection, intelligent video surveillance and other areas.

This paper is organized as follows: firstly, 2-D trajectory prediction algorithm is introduced; secondly, 3-D trajectory prediction algorithm is put forward; thirdly, the tracking effects based on3-D trace predicting are compared by simulation; at last, the author's work is summarized.
\end{abstract}

\section{2-D Trajectory Prediction}

During the process of target tracking, the position of the target is different in each frame. The position of the target in next frame is determined, according to the previous trajectory. The detailed matching search is carried around the 
prediction position, in order to improve the search speed. Trajectory of the target is continuous and differentiable. According to the theory of velocity and acceleration, a new motion polar coordinates equation can be obtained by three previous motion coordinates. Derivation process is as follows.

The principle of track prediction is shown in Fig. 1. Three previous motion coordinates are expressed as $\left(r_{1}, \theta_{1}\right),\left(r_{2}, \theta_{2}\right)$ and $\left(r_{3}, \theta_{3}\right)$. Prediction coordinates is expressed as $(r, \theta)$, where $r$ is the mold of vector and $\theta$ is the argument of vector in polar coordinates. According to the theory of velocity and acceleration, equation (3) can be derived by equation (1) and equation (2).

$$
v=v_{0}+a t
$$

$$
s=v_{0} t+\frac{1}{2} a t^{2}
$$

(2)

$$
s_{t}=v_{0} t+\frac{1}{2} a t^{2}-\left[v_{0}(t-1)+\frac{1}{2} a(t-1)^{2}\right]=v_{0}+a t-\frac{1}{2} a
$$

(3)

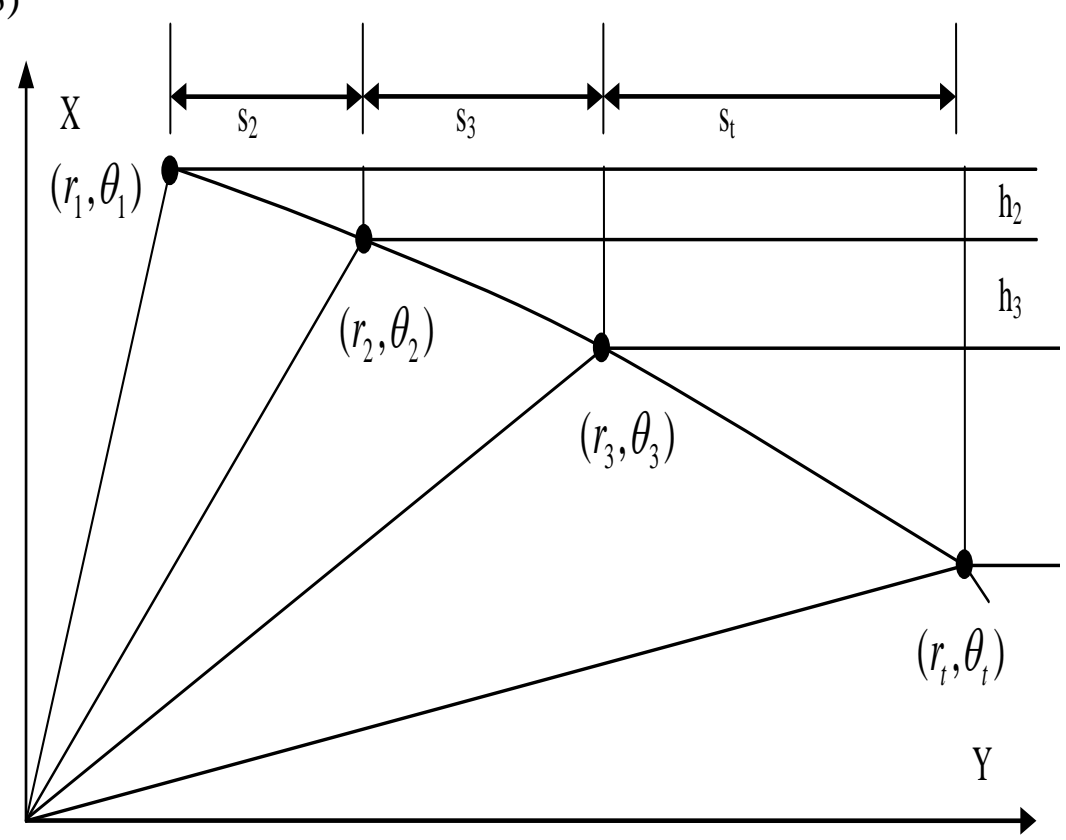

Fig. 1 Schematic diagram of 2-D trajectory prediction

Acceleration (a) is considered as a constant. Because time (t) is interval of two adjacent frames, it is considered as a constant. Equation (4) is derived. Through 
equation (5) and equation (6), equation (7) is derived. The moving distance of $\mathrm{X}$ direction in $\mathrm{t}$ frame is calculated by the first two frames.

$$
s_{t}=s_{t-1}+a
$$

(4)

$$
s_{t}-s_{t-1}=a
$$

(5)

$$
s_{t}-s_{t-1}=s_{t-1}-s_{t-2}=a
$$

(6)

$$
s_{t}=2 s_{t-1}-s_{t-2}
$$

$$
h_{t}=2 h_{t-1}-h_{t-2}
$$

(8)

The system of equations is composed of equation (9) and equation (10), and it is solved.

$$
r_{t} \operatorname{Sin} \theta_{t}=r_{t-1} \operatorname{Sin} \theta_{t-1}+s_{t}
$$

$$
r_{t} \operatorname{Cos} \theta_{t}=r_{t-1} \operatorname{Cos} \theta_{t-1}+h_{t}
$$

The argument of vector $\left(r_{t}\right)$ is calculated using iterative calculation method by equation (11). And the argument of vector $\left(\theta_{t}\right)$ is obtained by equation (12). Prediction coordinates $\left(r_{t}, \theta_{t}\right)$ is determined by equation (11) and equation (12).

$$
\begin{gathered}
r_{t}^{2}=r_{t-1}^{2}+\left(2 s_{t-1}-s_{t-2}\right) r_{t-1} \sin \theta_{t-1}+\left(2 s_{t-1}-s_{t-2}\right)^{2} \\
-\left(2 h_{t-1}-h_{t-2}\right) r_{t-1} \operatorname{Cos} \theta_{t-1}+\left(2 h_{t-1}-h_{t-2}\right)^{2} \\
\theta_{t}=\operatorname{arctg}\left(\frac{r_{t-1} \operatorname{Sin} \theta_{t-1}+2 s_{t-1}-s_{t-2}}{r_{t-1} \operatorname{Cos} \theta_{t-1}-2 h_{t-1}+h_{t-2}}\right)
\end{gathered}
$$

\section{3-D Trajectory Prediction}

Based on 2-D trajectory prediction algorithm, a mathematical model of 3-D trajectory prediction is established and proposed. The principle of 3-D track prediction is shown in Fig. 2. 
According to the space point $P(x, y, z)$, it can be described as $(r, \theta, \varphi)$ in the spherical coordinate system, too. Where $r$ is the distance between the origin $\mathrm{O}$ and the point $\mathrm{P}, \theta$ is the angle between directed line segment $\mathrm{OP}$ and $\mathrm{Z}$ axis, $\varphi$ is the angle between directed line segment $\mathrm{OM}$ and $\mathrm{X}$ axis. And point $\mathrm{M}$ is the projection of point $\mathrm{P}$ on the plane XOY. $s_{t}, h_{t}$ and $l_{t}$ in the first $\mathrm{t}$ frame is respectively the object displacement in the $\mathrm{X}$ direction, $\mathrm{Y}$ direction and $\mathrm{Z}$ direction. Prediction coordinates $(r, \theta, \varphi)$ is determined by equation (13), equation (14) and equation (15), where $r \in[0,+\infty), \varphi \in[0,2 \pi]$ and $\theta \in[0, \pi)$.

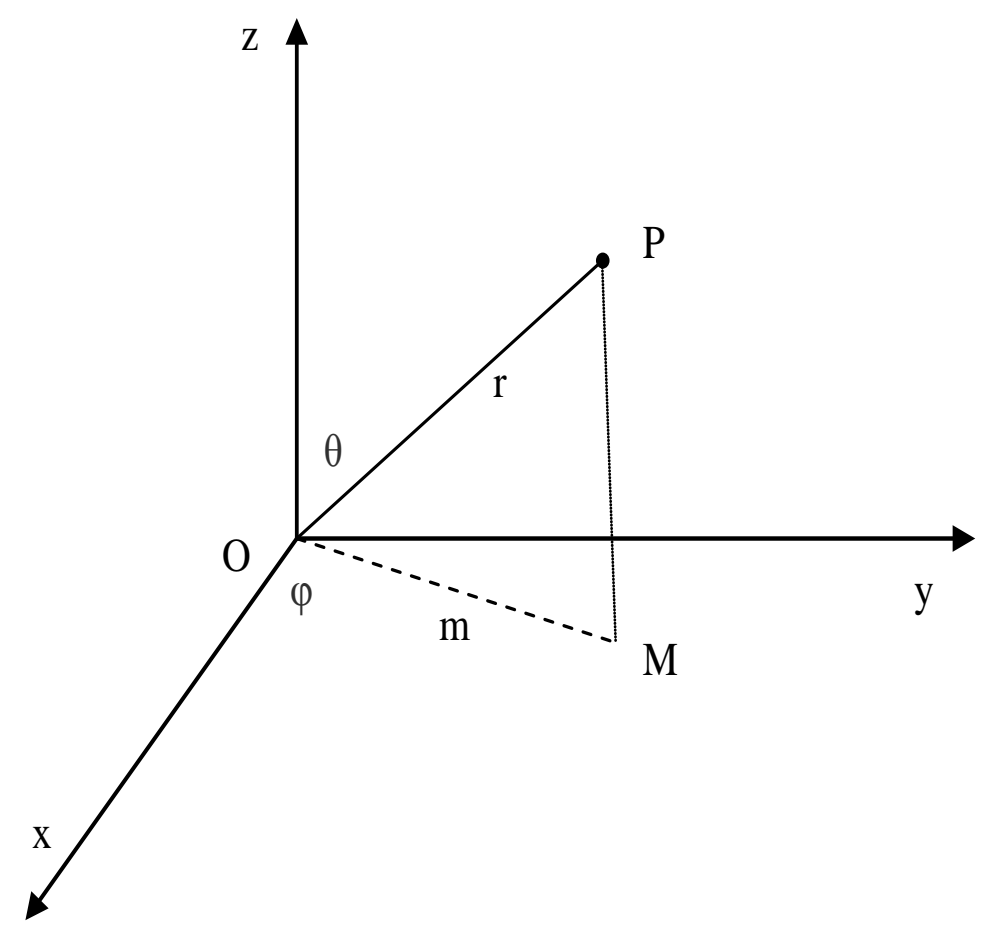

Fig. 2 Schematic diagram of 3-D trajectory prediction

$$
\begin{aligned}
r_{t}^{2}= & \left(r_{t-1} \operatorname{Sin} \theta_{t-1} \operatorname{Cos} \varphi_{t-1}+2 s_{t-1}-s_{t-2}\right)^{2}+ \\
& \left(r_{t-1} \operatorname{Sin} \theta_{t-1} \operatorname{Sin} \varphi_{t-1}+2 h_{t-1}-h_{t-2}\right)^{2}+ \\
& \left(r_{t-1} \operatorname{Cos} \theta_{t-1}+2 l_{t-1}-l_{t-2}\right)^{2}
\end{aligned}
$$




$$
\theta_{t}=\operatorname{arcCos}\left(\frac{r_{t-1} \operatorname{Sin} \theta_{t-1}+2 l_{t-1}-l_{t-2}}{r_{t}}\right)
$$

(14)

$$
\varphi_{t}=\operatorname{arctg}\left(\frac{r_{t-1} \operatorname{Sin} \theta_{t-1} \operatorname{Sin} \varphi_{t-1}+2 h_{t-1}-h_{t-2}}{r_{t-1} \operatorname{Sin} \theta_{t-1} \operatorname{Cos} \varphi_{t-1}+2 s_{t-1}-s_{t-2}}\right)
$$

(15)

\section{Test results}

The algorithm of 3-D trajectory prediction is simulated by MATLAB in the object tracking system. In the laboratory, the tracking experiments of a mouse are carried out. The mouse's position is predicted by 3-D trajectory prediction algorithm. Predictive values and actual values of target's position are shown in Table.1.The predictive and actual trajectories of moving target are compared in Fig. 3. Fitting curve of $r$ is shown in Fig. 3a, $\theta$ is shown in Fig. $3 b$ and $\varphi$ is

\begin{tabular}{|c|c|c|c|c|c|c|}
\hline \multirow{2}{*}{$\begin{array}{l}\text { Frame } \\
\text { s }\end{array}$} & \multicolumn{3}{|c|}{ Actual value } & \multicolumn{3}{|c|}{ Predictive value } \\
\hline & $\begin{array}{l}\text { r(pix } \\
\text { el) }\end{array}$ & $\begin{array}{c}\theta \text { (radia } \\
\text { n) }\end{array}$ & $\begin{array}{l}\varphi \text { (radian } \\
\text { ) }\end{array}$ & $\begin{array}{l}\text { r(pix } \\
\text { el) }\end{array}$ & $\begin{array}{c}\theta(\text { radia } \\
\text { n) }\end{array}$ & $\begin{array}{l}\varphi(\text { radian } \\
\text { ) }\end{array}$ \\
\hline 1 & 186.57 & 1.517 & 0.516 & & & \\
\hline 2 & 192.68 & 1.519 & 0.499 & & & \\
\hline 3 & 197.08 & 1.52 & 0.486 & & & \\
\hline 4 & 201.97 & 1.521 & 0.479 & 199.73 & 1.521 & 0.479 \\
\hline 5 & 206.41 & 1.522 & 0.468 & 207.32 & 1.523 & 0.477 \\
\hline 6 & 214.04 & 1.524 & 0.445 & 210.44 & 1.523 & 0.453 \\
\hline 7 & 221.7 & 1.526 & 0.433 & 225.02 & 1.526 & 0.412 \\
\hline 8 & 228.47 & 1.527 & 0.424 & 229.3 & 1.527 & 0.432 \\
\hline 9 & 233.95 & 1.528 & 0.414 & 234.35 & 1.528 & 0.418 \\
\hline 10 & 237.61 & 1.529 & 0.407 & 238.14 & 1.529 & 0.402 \\
\hline
\end{tabular}
shown in Fig.3c. 

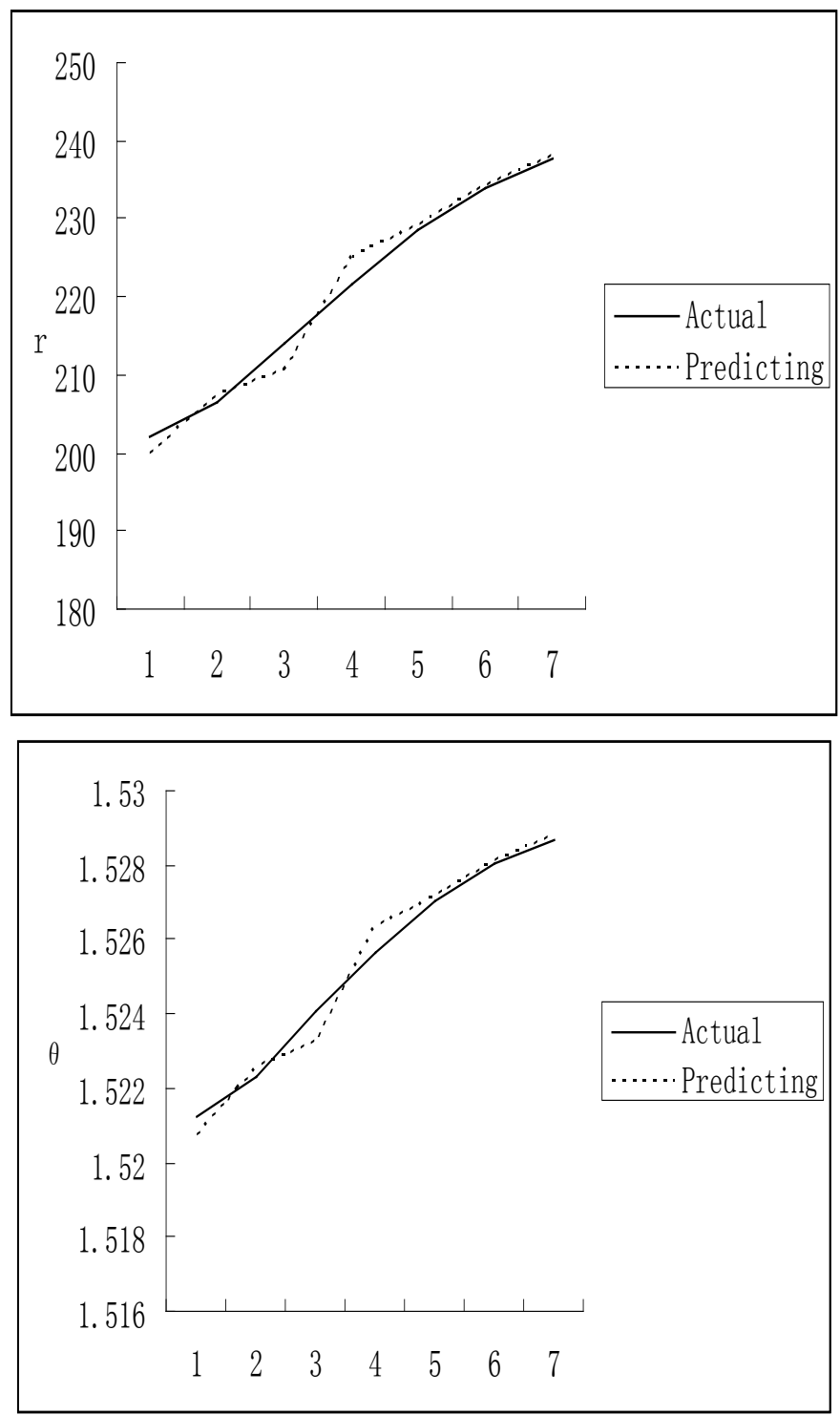
a. Fitting curve of $r$
b. Fitting curve of $\theta$ 


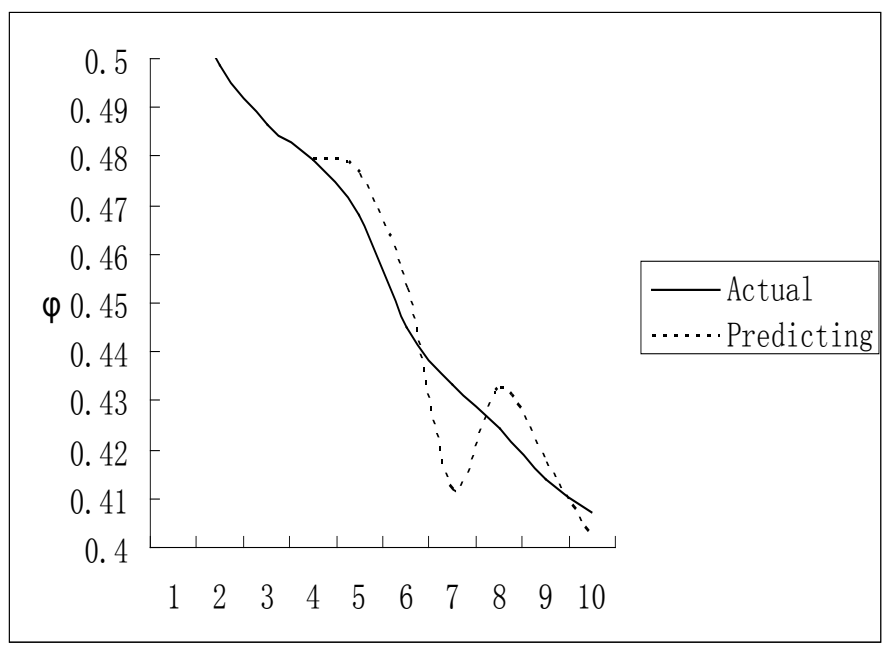

c. Fitting curve of $\varphi$

Fig. 3 Comparison of actual trajectory and prediction trajectory

\section{Conclusion}

A real-time predicting trace algorithm based 3-D is proposed and studied in this paper. And 3-D predicting trace algorithm is used in the target tracking system. Many tracking experiments are done by MALTAB simulation. The experiments show that this algorithm enhances the accurate rate of target tracking, and gets a satisfactory result.

\section{Acknowledgement}

This work was financially supported by scientific research fund of Jilin provincial education department (2014, the number: 302), Project name is "Research on the key technology of portable target recognition system based on multi-sensors fusion”.

\section{References}

[1] Jang D S, Choi H I, Active models for tracking moving objects. Pattern Recognition, Vol. 33 (2003), p. 1135-1139

[2] CHEN Jun-chao, LI Gang, Improved Anti-occlusion Tracking Algorithm of Multiple Moving Object. Journal of Chinese Computer Systems, Vol. 33(2012), p. 307-311. 
[3] NI Yu-de, LIU Ping and MA Yu-shen, An Improved Trajectory Prediction Algorithm Based on ADS-B Intent Information. Telecommunication Engineering, 2014.

[4] XUE Chen, ZHU Ming and LIU Chun-xiang, Review of tracking algorithms under occlusions. Chinese Journal of Optics and Applied Optics, Vol. 2 (2009), p. 388-392.

[5] GU Yu-feng, LIU Guo-dong, A moving target tracking algorithm under complicated scenes. Computer Technology and Its Applications, Vol. 38 (2012), p. $122-126$.

[6] Information

on

http://

en.cnki.com.cn/Article_en/CJFDTOTAL-DATE201402008.htm 